\title{
Linear and Non-Linear Indices of Heart Rate Variability in Chronic Heart Failure: Mutual Interrelationships and Prognostic Value
}

\author{
R Maestri ${ }^{1}$, GD Pinna ${ }^{1}$, P Allegrini ${ }^{2}$, R Balocchi $^{3}$, A Casaleggio $^{4}, \mathrm{G} \mathrm{D}^{\prime}$ Addio $^{1}, \mathrm{M} \mathrm{Ferrario}^{5}$,

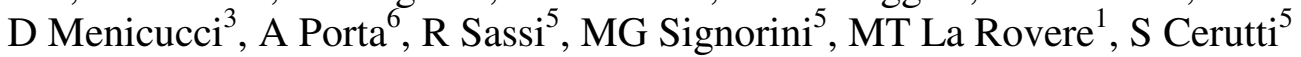 \\ ${ }^{1}$ Fondazione S Maugeri, IRCCS, Montescano and Telese, Italy \\ ${ }^{2}$ Istituto di Linguistica Computazionale, CNR, Pisa, Italy \\ ${ }^{3}$ Istituto di Fisiologia Clinica, CNR, Pisa, Italy \\ ${ }^{4}$ Istituto di Biofisica, CNR, Genova, Italy \\ ${ }^{5}$ Dipartimento di Bioingegneria, Politecnico di Milano, Italy \\ ${ }^{6}$ Dipartimento Scienze Precliniche, LITA Vialba, Università degli studi di Milano, Italy
}

\begin{abstract}
We computed 3 linear and 20 nonlinear HRV indexes on 24-h Holter recordings from 200 stable CHF patients

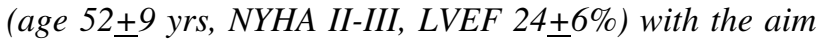
to assess i) the mutual interrelationships between these indexes and ii) their prognostic value towards cardiac death. We found high correlations between variables, with potential bias in fitting survival models. To overcome this problem a clustering procedure was used, obtaining 11 clusters. Cox analysis showed that seven clusters were significantly associated with the study outcome $(p<0.05)$ but, after adjustment for major clinical prognostic parameters, significance persisted only in 2 of them (both composed by nonlinear variables). Our results indicate that composite scores derived from nonlinear indices contain significant prognostic information independent of classical clinical predictors, highlighting the importance of non linear HRV parameters in prognostic stratification of $\mathrm{CHF}$ patients.
\end{abstract}

\section{Introduction}

A great deal of efforts have been made in recent years to find indices capable of providing prognostic information in chronic heart failure (CHF) patients, a pathology which represents a growing public health problem in industrialized countries, with high morbidity and mortality. Recent studies have consistently shown that some heart rate variability (HRV) measures obtained using linear techniques provide prognostic information in $\mathrm{CHF}$ patients, independent of clinical and functional parameters $[1,2]$.

It has been shown that nonlinear phenomena could play an important role in the genesis of HRV and several studies have been carried out to provide quantitative descriptors of complex heart rate dynamics; yet, the prognostic value of these parameters in heart failure has been scarcely investigated and only a few papers on this topic have been published up to now [3, 4].

In this study, carried out in cooperation by seven Italian groups, we computed three well established linear indices and twenty nonlinear indices of HRV derived from 24-hour Holter recordings. Our aim was to investigate the prognostic power of these indices as predictors of cardiac mortality among patients with $\mathrm{CHF}$ and to assess their mutual interrelationships.

The criteria for variable selection were to cover all main families of nonlinear parameters, keeping at the same time the number of variables as small as possible.

Due to the high risk of redundancy in information (multicollinearity), statistical analysis was performed using a clustering procedure to determine independent dimensions describing the overall set of variables.

\section{Methods}

\section{Subjects}

We studied 200 stable mild-to-moderate CHF patients in sinus rhythm admitted to the Heart Failure Unit of the Scientific Institute of Montescano (Italy). Inclusion criteria were stable clinical conditions during the last two weeks, 24 hour Holter recording analysable for at least half of the night-time (00:00-05:00 h) and half of the daytime (09:00-19:00 h). Within one week from the Holter recording, all patients underwent 2D echocardiography, cardiopulmonary exercise testing and routine blood tests.

The local ethics committee approved the study and all patients gave their informed consent. 


\section{Signal processing}

Holter recordings were processed using an Elatec system (ElaMedical, software release 3.0). Each beat was first automatically labelled by the Holter analysis software and then edited by an expert technician. Annotated RR time series were processed to correct for ectopic beats (linear interpolation). Finally, the annotated RR time series and the corrected ones were distributed to the investigators participating in the study. Each group performed his own analysis blind to clinical and event data and sent results back to the coordinating centre.

The number of candidate prognostic variables considered had to be limited according to the sample size of the study population and to the number of end-point events. Hence, the first step was the prospective selection of the indices to consider, basing the choice on previous studies and investigators' judgement. This led to a final selection of three linear indices and twenty nonlinear indices belonging to six major families, namely symbolic dynamics, entropy, empirical mode decomposition, fractality-multifractality, unpredictability or Poincarè plots.

The linear indices were: the standard deviation of all normal-to-normal RR intervals (SDNN), the power in the LF band (0.04-0.15 Hz) computed on all 5 minute segments and averaged (LFp) and the power in the VLF band (0.01-0.04 Hz) computed on all 5 minute segments and averaged (VLFp) [1, 2].

Three symbolic dynamics indices were computed: the first two by decomposing the RR time series into patterns lasting 3 beats and evaluating the percentage of patterns with one variation (1VP) and with 2 unlike variations (2UVP) respectively [5] and the third (BNI) was the binary coding nonrandomness index [6].

We considered three parameters derived from entropy: DELTA which is a measure of the long-range memory in RR time series [7], and BLZC and SampEn which are respectively binary Lempel-Ziv entropy [8] and sample entropy [9].

Four parameters were computed as representative of the fractality-multifractality family: the short-term detrended fluctuation analysis DFA [10], the Higuchi fractal dimension HFD [11], the slope of the power-law regression line of HRV (1/f slope) and the ratio between the width of the singularity multifractal spectrum and the same quantity after phase randomization SMFSr [12].

Two predictability indices were computed: normalized and not normalized unpredictability index based on conditional distribution (UPIn and UPI) [13].

We considered 4 parameters computed from the modes in which the original data are decomposed IMAI1, IMAI2, pLF2 and LFHFr. These parameters represent the ratio between the powers in LF1 (the mode closest to $0.1 \mathrm{~Hz}$ ) and $\mathrm{HF}$ (all modes with frequencies $>0.1 \mathrm{~Hz}$ ) bands, the ratio between the powers in LF2 (the first mode with frequency < than LF1) and HF bands, the power in band LF2 and the ratio between the powers in LF (LF1 + LF2) and HF bands. All parameters were computed on 5 minutes segments and then averaged [14].

Among quantitative indices provided by Poincare plots analysis, we considered the length of the 2D plots (LEN) and the radii of the 3D semi-ellipse of inertia along the $X$, Y and Z axes (RAD_X, RAD_Y, RAD_Z) [15].

Finally, NYHA class, left ventricular ejection fraction (LVEF), left ventricular end diastolic diameter (LVEDD), systolic arterial pressure (SAP) sodium (Na) and diagnosis (DIAG) were the clinical variables used as adjusting factors in survival analysis.

\section{Statistical analysis}

Skewed variables were properly transformed (logaritmic-, square-, square root- transformation) before analysis. The mutual interrelationship between variables was assessed by the Spearman correlation coefficient. To the purpose of reducing multicollinearity problems, cluster analysis was used. The condition for the clustering procedure was that the resulting cluster component (i.e. first principal component) had to explain at least $85 \%$ of the variance of the variables in the cluster. Cox proportional hazards regression analysis with and without adjustment for major clinical prognostic parameters was applied to each cluster component score and the bootstrap validation procedure used to check the stability of the models. Verification of the proportional hazards assumption was performed plotting the smoothed scaled Schoenfeld residuals.

\section{Results}

Major clinical characteristics of the patients are given in table 1 . Seventy five patients (37\%) experienced an event during the follow-up period of $24 \pm 13$ months.

Variables within families tended to be correlated, but we observed high correlations also between variables from different families, including linear and nonlinear groups. The couples of variables showing a Spearman correlation coefficient modulus $\geq 0.8$ are listed in table 2 . Four nonlinear parameters (pLF2, LEN, RAD_Z, UPI) were so strongly correlated with linear variables that we discarded them from subsequent clustering procedure.

We obtained eleven clusters, each represented by a cluster component score, with a total variance explained of $91 \%$. The three linear variables resulted grouped in a single cluster, three clusters were composed of more than one nonlinear variable while the remaining were single nonlinear variable clusters. Cox proportional hazard regression analysis showed that seven cluster components were significantly associated $(\mathrm{p}<0.05)$ with the study outcome. 
Table 1. Clinical characteristics of the population studied. LVEF: Left ventricular ejection fraction, VPC: ventricular premature contractions, NSVT: non sustained ventricular tachycardia. Data are expressed as median $\left(25^{\text {th }}\right.$ percentile, $75^{\text {th }}$ percentile $)$.

\begin{tabular}{lc}
\hline \hline Age, yrs & $54(47,58)$ \\
Male, \% & 87 \\
NYHA Class II-III, \% & 88 \\
Cause & \\
$\quad$ Ischemic, \% & 50 \\
$\quad$ Idiopathic, \% & 45 \\
$\quad$ Other, \% & 5 \\
LVEF, \% & $23(19,28)$ \\
VPCs/h, n & $13(3,47)$ \\
NSVT, \% & 37 \\
\hline \hline
\end{tabular}

Table 2. Couples of variables with a Spearman correlation coefficient modulus $\geq 0.8$. $1 \mathrm{~g}$ : logarithmic transformation, sqr: square root transformation, sq: square transformation

\begin{tabular}{|c|c|c|}
\hline & & $\begin{array}{c}\text { Spearman } \\
\mathrm{R} \\
\end{array}$ \\
\hline $\lg (\mathrm{VLFp})$ & $\lg (\mathrm{LFp})$ & 0.87 \\
\hline $\lg (\mathrm{VLFp})$ & $\lg (\mathrm{pLF} 2)$ & 0.92 \\
\hline $\lg (\mathrm{LFp})$ & $\lg (\mathrm{pLF} 2)$ & 0.90 \\
\hline $\lg (\mathrm{LFp})$ & $\lg (\mathrm{UPI})$ & 0.82 \\
\hline $\lg (\mathrm{SDNN})$ & LEN & 0.93 \\
\hline $\lg (\mathrm{SDNN})$ & $\lg (\mathrm{RAD} Z \mathrm{Z})$ & 0.97 \\
\hline $\lg (\mathrm{SDNN})$ & $\lg (\mathrm{pLF} 2)$ & 0.80 \\
\hline LEN & $\lg (\mathrm{RAD} Z \mathrm{Z})$ & 0.89 \\
\hline $\lg ($ RAD_Z) & $\lg (\mathrm{pLF} 2)$ & 0.81 \\
\hline sqr(IMAI2) & UPIn & -0.82 \\
\hline sqr(IMAI2) & sq(DFA) & 0.81 \\
\hline $\lg (\mathrm{pLF} 2)$ & $\lg (\mathrm{UPI})$ & 0.83 \\
\hline $\lg (\mathrm{LFHFr})$ & sqr(IMAI1) & 0.87 \\
\hline sqr(IMAI1) & UPIn & -0.89 \\
\hline sqr(IMAI1) & HFD & -0.86 \\
\hline sqr(IMAI1) & sq(DFA) & 0.89 \\
\hline UPIn & sq(DFA) & -0.92 \\
\hline HFD & sq(DFA) & -0.82 \\
\hline
\end{tabular}

Bootstrap validation substantially confirmed reported results and the proportional hazard assumption was satisfied by all models.

After adjustment for clinical prognostic variables, statistical significance persisted in only two clusters: cluster 1 composed by IMAI2, LFHFr, IMAI1, UPIn and DFA, and cluster 6 composed by 1VP only. Table 3 summarizes the clusters composition and the association with outcome before and after adjustment for clinical variables.

Table 3. Clusters composition and respective association with cardiac mortality in univariate analysis and after adjustment for clinical correlates.

\begin{tabular}{|c|c|c|c|}
\hline & & $\begin{array}{c}\mathrm{p} \\
\text { univariate } \\
\end{array}$ & $\begin{array}{c}\mathrm{p} \\
\text { adjusted }\end{array}$ \\
\hline CLUS1 & $\begin{array}{c}\text { sqr(IMAI2) } \\
\lg (\mathrm{LFHFr}) \\
\text { sqr(IMAI1) } \\
\text { UPIn } \\
\text { sq(DFA) } \\
\end{array}$ & 0.0002 & 0.03 \\
\hline CLUS2 & $\begin{array}{c}\lg (\text { VLFp }) \\
\lg (\text { LFp }) \\
\lg (\text { SDNN })\end{array}$ & 0.0006 & 0.30 \\
\hline CLUS3 & sqr(SMFSr) & 0.30 & 0.13 \\
\hline CLUS4 & 1/f slope & 0.21 & 0.79 \\
\hline CLUS5 & $\begin{array}{c}\text { sq(BLZC) } \\
\text { HFD }\end{array}$ & 0.0004 & 0.18 \\
\hline CLUS6 & $\mathrm{sq}(1 \mathrm{VP})$ & 0.002 & 0.01 \\
\hline CLUS7 & $\lg ($ RAD_Y $)$ & 0.066 & 0.17 \\
\hline CLUS8 & sq(Delta) & 0.017 & 0.43 \\
\hline CLUS9 & $\lg ($ SampEn $)$ & 0.138 & 0.29 \\
\hline CLUS10 & $\begin{array}{c}\lg (\mathrm{BNI}) \\
\operatorname{sqr}(2 \mathrm{UVP}) \\
\end{array}$ & 0.035 & 0.80 \\
\hline CLUS11 & $\lg ($ RAD_X $)$ & 0.0017 & 0.17 \\
\hline
\end{tabular}

\section{Discussion and conclusions}

This study shows that in mild-to-moderate CHF patients the information conveyed by nonlinear indices of 24-hour HRV has a prognostic value independent of major clinical predictors known so far. The cluster composed by the combination of linear indices, though strongly significant in univariate analysis, lost its power when adjusted for clinical variables. This finding is not totally unexpected, since spectral indices of HRV have always shown to be more powerful predictors in $\mathrm{CHF}$ after dichotomous categorisation, while in the present study they were considered as continuous variables.

Among nonlinear variables, out of the 10 clusters considered, in only two of them significance persisted after adjustment. The first of these clusters (CLUS1) contained only the symbolic dynamics variable $1 \mathrm{VP}$. It 
has been recently shown that variables of this type are capable to detect the changes in sympathetic and vagal modulation elicited by experimental and pharmacological interventions [16]. The second cluster (CLUS6) was composed by 5 variables: 3 from the empirical mode decomposition family, one from predictability and one from the fractality-multifractality class. All the variables in CLUS1 and CLUS6 are related to short-term temporal scales, pointing out the importance of short-term correlation properties in our patients.

Although computed using markedly different algorithms, some nonlinear indexes of HRV in CHF patients contain highly correlated information, and some others are highly correlated with classical linear indexes. Even though this finding is not new in some cases (e.g. the strong association between Poincarè plots parameter LEN and linear index SDNN has already been observed and studied), further research is needed to investigate the possible mechanisms underlying these relationships.

In conclusion, our findings indicate that methods derived from nonlinear system theory can provide significant prognostic information independent of classical clinical predictors and additional to standard linear HRV measures, and highlights the importance of non linear HRV parameters in prognostic stratification of CHF patients.

\section{References}

[1] La Rovere MT, Pinna GD, Maestri R, Mortara A, Capomolla S, Febo O, Ferrari R, Franchini M, Gnemmi M, Opasich C, Riccardi PG, Traversi E, Cobelli F. Short-term heart rate variability strongly predicts sudden cardiac death in chronic heart failure patients. Circulation 2003; 107 (4): 565-570.

[2] Guzzetti S, La Rovere MT, Pinna GD, Maestri R, Borroni E, Porta A, Mortara A, Malliani A. Different spectral components of $24 \mathrm{~h}$ heart rate variability are related to different modes of death in chronic heart failure. Eur Heart J 2005; 26 (4): 357-362.

[3] Makikallio TH, Huikuri HV, Hintze U, Videbaek J, Mitrani RD, Castellanos A, Myerburg RJ, Moller M; DIAMOND Study Group (Danish Investigations of Arrhythmia and Mortality ON Dofetilide). Fractal analysis and time- and frequency-domain measures of heart rate variability as predictors of mortality in patients with heart failure. Am J Cardiol 2001; 87: 178-182.

[4] Ho KK, Moody GB, Peng CK, Mietus JE, Larson MG, Levy D, Goldberger AL. Predicting survival in heart failure case and control subjects by use of fully automated methods for deriving nonlinear and conventional indices of heart rate dynamics. Circulation 1997; 96 (3): 842-848.

[5] Porta A, Guzzetti S, Montano N, Furlan R, Pagani M, Malliani A, Cerutti S. Entropy, entropy rate, and pattern classification as tools to typify complexity in short heart period variability series. IEEE Trans Biomed Eng 2001; 48
(11): 1282-1291.

[6] Yang AC, Hseu SS, Yien HW, Goldberger AL, Peng CK. Linguistic Analysis of the human heartbeat using frequency and rank order statistics. Phys Rev Lett 2003; 90 (10): 108103.

[7] Allegrini P, Grigolini P, Hamilton P, Palatella L, Raffaelli G. Memory beyond memory in heart beating, a sign of a healthy physiological condition. Physical Review E, Stat Nonlin Soft Matter Phys 2002; 65: 041926.

[8] Ferrario M, Signorini M.G., Cerutti S., "Complexity Analysis of 24 Hours Heart Rate Variability Time Series", Proc. of the EMBC 04 Conference, IEEE Press.

[9] Richman JS, Moorman JR. Physiological time-series analysis using approximate entropy and sample entropy. Am J Physiol Heart Circ Physiol 2000; 278: H2039-2049.

[10] Peng CK, Havlin S, Stanley HE, Goldberger AL. Quantification of scaling exponents and crossover phenomena in nonstationary heartbeat time series. CHAOS 1995; 5: 82-87.

[11] Accardo A, Affinito M, Carrozzi M, Bouquet F. Use of the fractal dimension for the analysis of electroencephalographic time series. Biol Cybern 1997; 77 (5): 339-350.

[12] Ivanov PC, Amaral LA, Goldberger AL, Havlin S, Rosenblum MG, Struzik ZR, Stanley HE. Multifractality in human heartbeat dynamics. Nature 1999; 399 (6735): 461465.

[13] Porta A, Baselli G, Guzzetti S, Pagani M, Malliani A, Cerutti S. Prediction of short cardiovascular variability signals based on conditional distribution. IEEE Trans Biomed Eng 2000; 47 (12): 1555-1564.

[14] Balocchi R, Menicucci D, Santarcangelo E, Sebastiani L, Gemignani A, Ghelarducci B, Varanini M. Deriving the respiratory sinus arrhythmia from the heartbeat time series using empirical mode decomposition. Chaos, Solitons and Fractals 2004; : 171-177.

[15] D'Addio G, Pinna GD, La Rovere MT, Maestri R, Furgi G, Rengo F. Prognostic Value Of Poincarè Plot Indexes In Chronic Heart Failure Patients. Computers in Cardiology, IEEE Computer Society Press 2001; 28: 57-60.

[16] Guzzetti S, Borroni E, Garbelli PE, Ceriani E, Della Bella P, Montano N, Cogliati C, Somers VK, Mallani A, Porta A. Symbolic dynamics of heart rate variability: a probe to investigate cardiac autonomic modulation.. Circulation 2005; 112 (4): 465-470.

Address for correspondence

Roberto Maestri

S. Maugeri Foundation, IRCCS,

Scientific Institute of Montescano, 27040 Montescano (Pavia)

Italy

e-mail: rmaestri@fsm.it 